\title{
Genomic bases underlying the adaptive radiation of core landbirds
}

\author{
Yonghua Wu ${ }^{1 *} \mathbb{D}$, Yi Yan' ${ }^{1}$ Yuanqin Zhao', Li Gu' , Songbo Wang ${ }^{2}$ and David H. Johnson ${ }^{3 *}$
}

\begin{abstract}
Background: Core landbirds undergo adaptive radiation with different ecological niches, but the genomic bases that underlie their ecological diversification remain unclear.

Results: Here we used the genome-wide target enrichment sequencing of the genes related to vision, hearing, language, temperature sensation, beak shape, taste transduction, and carbohydrate, protein and fat digestion and absorption to examine the genomic bases underlying their ecological diversification. Our comparative molecular phyloecological analyses show that different core landbirds present adaptive enhancement in different aspects, and two general patterns emerge. First, all three raptorial birds (Accipitriformes, Strigiformes, and Falconiformes) show a convergent adaptive enhancement for fat digestion and absorption, while non-raptorial birds tend to exhibit a promoted capability for protein and carbohydrate digestion and absorption. Using this as a molecular marker, our results show relatively strong support for the raptorial lifestyle of the common ancestor of core landbirds, consequently suggesting a single origin of raptors, followed by two secondary losses of raptorial lifestyle within core landbirds. In addition to the dietary niche, we find at temporal niche that diurnal birds tend to exhibit an adaptive enhancement in brightlight vision, while nocturnal birds show an increased adaption in dim-light vision, in line with previous findings.
\end{abstract}

Conclusions: Our molecular phyloecological study reveals the genome-wide adaptive differentiations underlying the ecological diversification of core landbirds.

Keywords: Gene capture, Convergent evolution, Raptor, Digestion, Ancestral trait reconstruction

\section{Background}

Core landbirds (Telluraves) is the recently defined largest clade in Neoaves, including raptors, woodpeckers, parrots, and songbirds [1-3]. Core landbirds adapt to diverse environments with different phenotypes related to diets, diel activity patterns, language and hearing, etc. [4]. Adapting to diverse environments, different core landbirds may have been subjected to divergent selections, whereas for ecologically similar taxa, for instance, three birds of prey (Accipitriformes, Strigiformes, and

\footnotetext{
*Correspondence: wuyh442@nenu.edu.cn; djowl@aol.com

1 School of Life Sciences, Northeast Normal University,

Changchun 130024, China

${ }^{3}$ Global Owl Project, 6504 Carriage Drive, Alexandria, VA 22310, USA

Full list of author information is available at the end of the article
}

Falconiformes), which share many raptorial-lifestyle characteristics, similar selections may have occurred between them [5]. Despite their ecological similarities, phylogenetic analyses show that the three birds of prey are relatively distantly related, and it remains unknown whether their similar raptorial lifestyles are a result of single origin [2,3] or multiple origin [1].

The Avian Phylogenomics Project evaluates the genomic evolution of 48 avian species within the class Aves with relatively little detailed analyses of genomic difference among avian subgroups, such as core landbirds [6]. Other comparative genomic or transcriptomic studies related to core landbirds are restricted to specific taxa [7-13] or specific genes related to vision and digestion [14-16]. A recent study of 16 genomes of birds of prey reveals their shared genetic adaptation underlying their 
anatomical structures and sensory, muscle, circulatory, and respiratory systems related to a raptorial lifestyle [5]. Despite these efforts, a more comprehensive study with a sufficient sampling across both different taxa and functionally different genes is needed to better elucidate the integrative evolution of core landbirds in adapting to diverse ecological environments.

The recent development of a molecular phyloecological (MPE) approach, which employs a comparative phylogenetic analysis of biologically meaningful genes, has been demonstrated to have power to reveal the genetic bases that underlie phenotypic differentiations and enable ancestral trait reconstruction $[17,18]$. In light of the MPE approach, we in this study use target enrichment sequencing to obtain gene sequences to examine the genetic bases that underlie the ecological diversification of core landbirds. Target enrichment sequencing has been widely used in gene capture across species [19-22]. Our comparative analyses show that different core landbird taxa exhibit taxon-specific adaptations, whereas ecologically similar taxa present somewhat similar evolutionary adaptations. Our study reveals the genomic bases underlying the adaptive radiation of core landbirds and provides insights into the evolutionary history of their ecological diversification.

\section{Results}

We used target enrichment sequencing to obtain the coding sequences of 308 genes related to vision, hearing, language, temperature sensation, beak shape, taste transduction, and carbohydrate, protein and fat digestion and absorption (Additional file 1, Table S1; Additional file 13) from 46 core landbird taxa with an address on the sampling of birds of prey (Fig. 1, Additional file 2: Table S2), for which there are only sparse genome data available thus far. For the target enrichment sequencing of our focal 308 genes, we designed baits based on the coding sequences of these genes from multiple divergent species (e.g., falcon, eagle and owl), which has been demonstrated to be a highly effective strategy for increasing capture efficiency [22]. We then employed the same set of baits to capture and sequence our target genes across the 46 taxa using the MYbaits target enrichment system (MYcroarray, Arbor Biosciences) (Additional file 2: Table S2). The average number of reads sequenced across our 46 taxa was $4,716,979.58$, the average total base pair yield was $1,178.65 \mathrm{Mb}$, and the average sequencing coverage and sequencing depth across the 46 taxa were $94.53 \%$ and $272.76 \mathrm{X}$, respectively, while 13 genes showed a sequencing coverage of $<60 \%$, and 17 genes showed a sequencing depth of $<10 \mathrm{X}$. Raw reads were filtered, and de novo assembled, and coding sequences were obtained for subsequent adaptive evolution analyses. We followed the MPE approach and used likelihood ratio tests based on branch and branch-site models implemented in the Codeml program of PAML [23] to identify positively selected genes (PSGs) along branches leading to diverse core landbird taxa. In addition to PAML, we employed the program RELAX [24] to examine the selection strength changes (e.g., intensified or relaxed) of genes for our focal taxa.

\section{Selection analyses of the genes related to senses, language, beak and temperature}

To examine the molecular bases of phenotypic differentiations of core landbird taxa, we initially constructed a dataset by combining the captured coding sequences of our 46 taxa with the sequence data of 33 core landbird species available from GenBank (Fig. 1, Additional file 3: Table S3), referred to as the core landbird dataset hereafter. Based on the core landbird dataset, we firstly used PAML to examine the adaptive evolution of the 33 genes involved in the phototransduction pathway (Fig. 2, Additional files 4, 5: Tables S4, 5), which have been used as the molecular markers to determine the diel activity patterns of birds and mammals $[17,18]$. We found five PSGs (GRK1, LWS, RH2, SWS2, and ARR3) along the branch leading to Strigiformes (owls), which are mainly crepuscularly and nocturnally active. GRK1 is a rod-expressed gene and is involved in the inactivation of activated rhodopsin, contributing to photoresponse recovery in dimlight conditions. LWS $R H 2$, and SWS2, respectively, encode red-, green-, and blue-sensitive cone opsins, and their positive selections in owls are linked to spectral tuning to maximize light abortion under crepuscular conditions, as previously evidenced [16]. ARR3 is involved in the inactivation of cone opsins for photoresponse recovery. Moreover, we did not find any captured sequences of violet- or ultraviolet-sensitive opsin gene (SWS1) from all 28 owl species examined, and this was consistent with previous studies that consistently show the absence of the gene expression or sequence of SWS1 in owls $[8,9,14,16]$. In addition to Strigiformes, we found PSGs along the branch leading to Coraciimorphae and Passeriformes, both of which are generally diurnal $[4,17]$. For Coraciimorphae, two PSGs (CNGB3 and SLC24A2) were found. CNGB3 and SLC24A2 are coneexpressed and are respectively involved in photoresponse and photoresponse recovery in bright light conditions. For Passeriformes, five PSGs (GNAT2, LWS, GUCA1C, $S L C 24 A 2$, and $R C V R N$ ) were detected, and almost all of them were cone-expressed, involved in photoresponse and photoresponse recovery in bright light conditions. Finally, we also found two rod-expressed PSGs (GRK1 and SLC24A1) along branch A (Fig. 1), representing the 


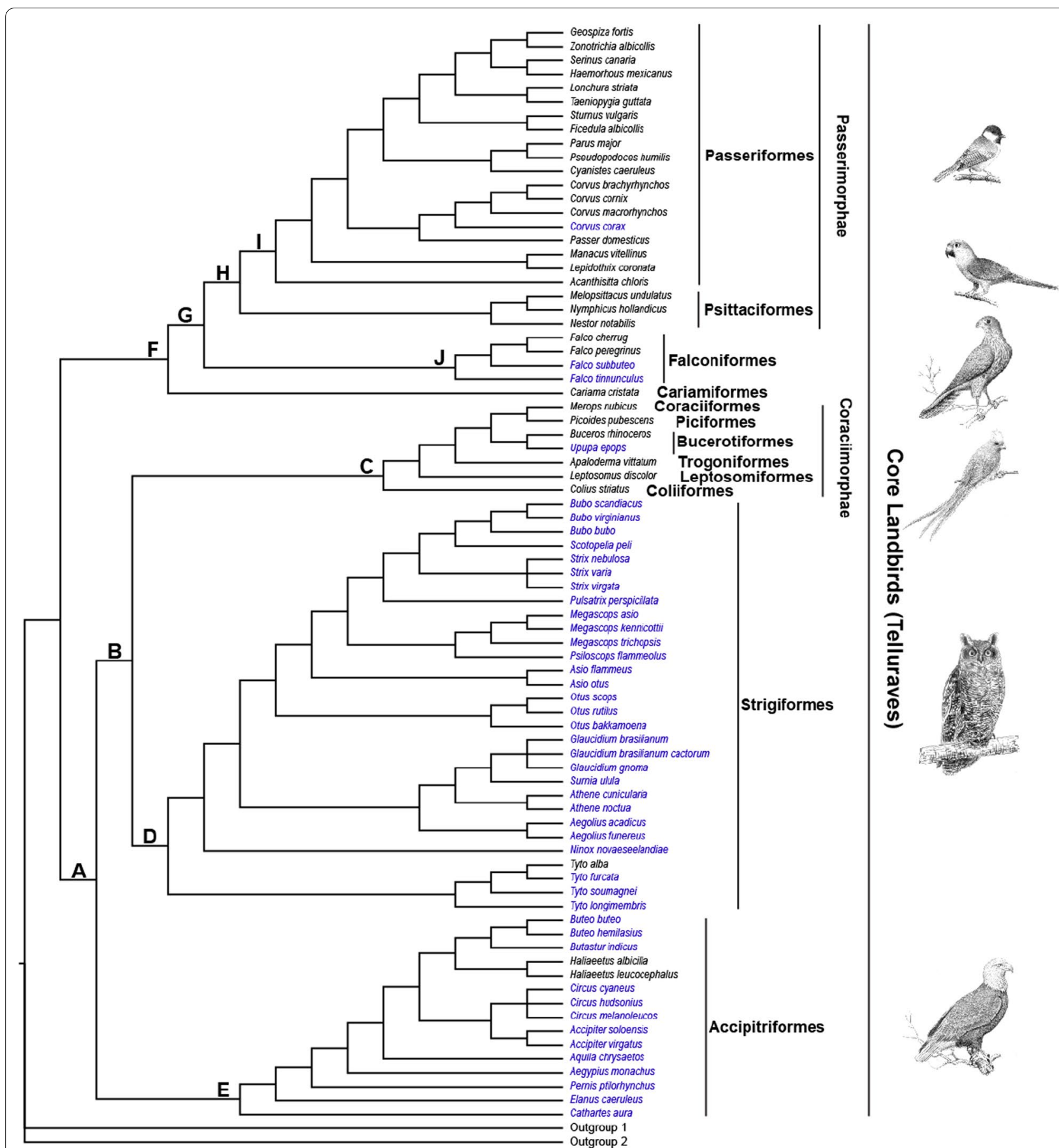

Fig. 1 Phylogenetic relationships of core landbird species used in this study. Phylogenetic relationships among species follow published studies [2, 17, 59, 60, 64-73] and the Tree of Life Web Project (http://tolweb.org/tree/). The species under gene capture in this study are shown in blue, and the species with gene sequences cited from GenBank are shown in black. The letters (A-J) show the branches under positive selection analyses based on core landbird dataset in this study

common ancestor of Accipitriformes, Strigiformes and Coraciimorphae.

We subsequently examined the positive selection of 95 hearing-related genes, and relevant gene functions were referred to OMIM in NCBI. Our results showed that Coraciimorphae and Passeriformes showed the highest number of PSGs among the diverse core landbird taxa examined (Additional files 4, 5: Tables S4-5). For 


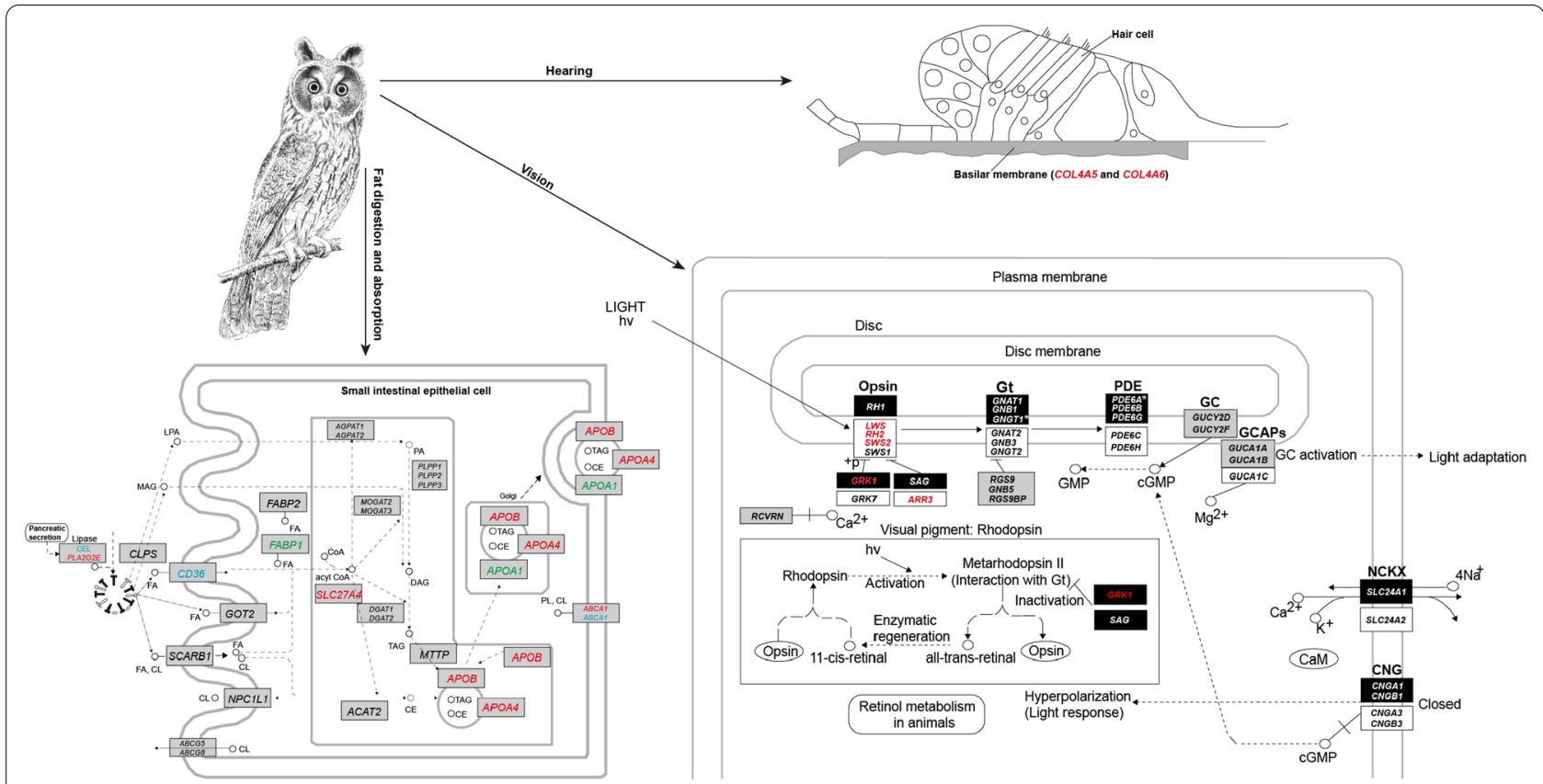

Fig. 2 Positively selected and selectively intensified genes related to vision, hearing, and fat digestion and absorption in Strigiformes (highlighted in red). For convenience, the positively selected and/or selectively intensified genes of fat digestion and absorption found in the branches related to Accipitriformes (green) and Falconiformes (blue) are also shown. The top right shows the cross section of inner ear (cochlea), and the lower right shows the phototransduction pathway (according to KEGG pathway map 04744), and the lower left shows the fat digestion and absorption pathway (according to KEGG pathway map 04975). For the phototransduction pathway, the genes that are involved in rods, cones, and both are shown as dark rectangles, white rectangles, and gray rectangles, respectively [16, 18]. *Represents two lost rod-expressed genes, GNGT1 and PDE6A, in both reptiles and birds based on previous studies $[16,18]$

Coraciimorphae, five PSGs (ATP2B2, MYO7A, PRPS1, $S L C 9 A 9$, and $U S H 2 A$ ) were found. ATP2B2 plays a critical role in intracellular calcium homeostasis, and its mutation is linked to deafness and imbalance. MYO7A plays a role in the development and maintenance of stereocilia, which is critical for converting sound waves to nerve impulses. PRPS1 encodes an enzyme called phosphoribosyl pyrophosphate synthetase 1 , and its mutations have been linked to a type of nonsyndromic X-linked sensorineural deafness. USH $2 \mathrm{~A}$ encodes usherin and is an important component of basement membranes in the inner ear and in the retina. SLC9A9 encodes a sodium/ proton exchanger and may play an important role in maintaining cation homeostasis. For Passeriformes, five PSGs (CRYM, FKBP14, PTPRQ, WNT8A, and MYO7A) were detected. $C R Y M$ encodes a crystallin protein, and its mutations may cause autosomal dominant non-syndromic deafness. FKBP14 gene encodes a protein called FKBP prolyl isomerase 14, and its mutations are linked to hearing loss. PTPRQ encodes a member of the type III receptor-like protein-tyrosine phosphatase family, and its mutations may cause autosomal recessive deafness. WNT8A encodes a protein of the WNT gene family and may be involved in the development of early embryos and germ cell tumors. In addition to Coraciimorphae and Passeriformes, positively selected hearing-related genes were found in other taxa. Notably, COL4A5 and COL4A6 were found to be under positive selection in owls (Fig. 2, Additional file 5: Table S5). The two genes encode two of the six subunits of type IV collagen, the major structural component of basement membranes in the inner ear. Mutations of the two genes are associated with hearing loss and the severity of deafness. For Falconiformes (falcons), two PSGs (MYO7A and SLC17A8) were detected; $M Y O 7 A$ is involved in the development and maintenance of stereocilia, and SLC17A8 encodes a vesicular glutamate transporter. We also detected one positively selected gene, $T M C 1$, along branch $\mathrm{H}$, which represents the common ancestor of Passeriformes and Psittaciformes. TMC1 is required for the normal functioning of cochlear hair cells, and its mutations have been associated with progressive postlingual hearing loss and profound prelingual deafness.

We also analyzed the positive selection of the genes related to taste, language, beak shape, and temperature sensation (Additional files 4, 5: Tables S4-5). Among the 43 taste-related genes analyzed, several PSGs were found in different taxa. Though these PSGs found are related 
to taste transduction, only one positively selected gene, SCNN1A, which was found along the branch leading to Accipitriformes, is relatively clearly related to taste (salt) perception; other PSGs, such as PDE1C, GNB3, and ENTPD2, are found to be widely expressed in many other tissues as well (please see OMIM in NCBI for details). We also examined the positive selection of 24 languageand/or speech-related genes, and our results showed that $R O B O 1$ in owls, $D C D C 2$ in falcons, and FOXP2 in the common ancestor of Falconiformes and Passerimorphae were found to be under positive selection. $R O B O 1$ is involved in core trait underpinning language acquisition, $D C D C 2$ encodes a doublecortin domain-containing family member and is linked to dyslexia, and FOXP2 encodes a member of the forkhead/winged-helix (FOX) family of transcription factors and is required for the proper development of speech and language. Finally, we analyzed the positive selection of 15 beak shape- related genes and 15 temperature sensation-related genes. For the beak shaperelated genes, we found only one positively selected gene, $D K K 3$, which showed a positive selection signal in owls. $D K K 3$ plays a role in the development related to beak depth and length. For the temperature sensation-related genes, three PSGs were detected, including one cold sensor (TRPA1) in owls, one warm sensor (TRPM2) in falcons, and one warm sensor (TRPV4) in the common ancestor of owls and their sister taxon, Coraciimorphae.

\section{Selection analyses of digestive system-related genes}

We used PAML to examine the adaptive evolution of the 84 genes that are involved in the carbohydrate, protein and fat digestion and absorption pathways to determine the possible selection differences between core landbird taxa. Our initial analyses using PAML showed that almost all examined branches exhibited positive selection signals in the genes related to carbohydrate and/or protein digestion and absorption to some extent, with the exception of two raptor-related branches (branches G and D, Fig. 1), which also showed positive selection on the genes related to fat digestion and absorption, including one gene (CEL) along the common ancestor branch (branch $\mathrm{G}$ ) of Falconiformes and Passerimorphae, and three genes (APOA4, $A P O B$, and SLC27A4) along the branch (branch D) leading to owls (Additional files 4, 5, 6: Tables S4-6). To further examine whether the positive selections of the fat digestion and absorption-related genes occurred uniquely along raptor-related branches, we then respectively evaluated the relative selection intensity changes of the 84 genes along the branches leading to three birds of prey and their sister taxa compared to their common ancestor branches using program RELAX [24] (Fig. 1, Additional file 7: Table S7). Intriguingly, our results showed that all three birds of prey showed selectively intensified genes related to fat digestion and absorption (Fig. 2), including CD36 in Falconiformes (branch J compared to branch G), $A B C A 1$ in Strigiformes (branch D compared to branch $\mathrm{B}$ ), and $A P O A 1$ in Accipitriformes (branch E compared to branch A), though Falconiformes and Strigiformes showed a selection intensification on the genes related to carbohydrate and/or protein digestion and absorption as well (Additional files 6, 7: Tables S6-7). Unlike the three birds of prey, which almost consistently exhibited the selection intensification of the genes related to fat digestion and absorption to some extent, their sister taxa, Coraciimorphae and Passerimorphae, showed somewhat variable results (Additional file 6, 7: Tables S6-7). Especially for Coraciimorphae (branch $\mathrm{C}$ ), almost all detected genes showed a relative selection relaxation compared to the common ancestor branch (branch B) of Coraciimorphae and Strigiformes, and these genes were found to be mainly related to fat and protein digestion and absorption. For Passerimorphae (branch $\mathrm{H})$, four genes (CD36, $C P B 2, C T R L$ and $P R K C B$ ), which are involved in fat, protein and carbohydrate digestion and absorption, showed a relative intensified selection compared to the common ancestor branch (branch G) of Passerimorphae and Falconiformes, while two genes ( $L C T$ and PIK3CD) related to carbohydrate digestion and absorption showed a relative selection relaxation. Taken together, it seems that all three birds of prey showed a relative intensified selection for fat digestion and absorption to some extent, while their non-raptorial relatives showed somewhat variable results.

Our RELAX results described above revealed the selection differences of the digestive system-related genes between raptors and non-raptorial birds, showing that all three birds of prey were characterized by an intensified selection for fat digestion and absorption. In light of this finding, we subsequently examined the selection intensity changes of these digestive system-related genes for the common ancestor of core landbirds studied, which is considered to be an apex predator [2,3]. To this end, we constructed a new dataset, which included largely the full-length coding sequences of these digestive system-related genes from all avian taxa with gene sequences available from GenBank (Fig. 3, Additional file 8: Table S8), referred to as the whole avian dataset hereafter, considering that our core landbird dataset used involved only a few outgroups of core landbirds (Fig. 1), and that its gene sequences used were relatively short. Based on the whole avian dataset, we examined the relative selection intensity changes of our focal genes along the common ancestor branch (branch L) of core landbirds compared to the common ancestor branch (branch M) of core landbirds and their sister taxa, waterbirds (Fig. 3). 


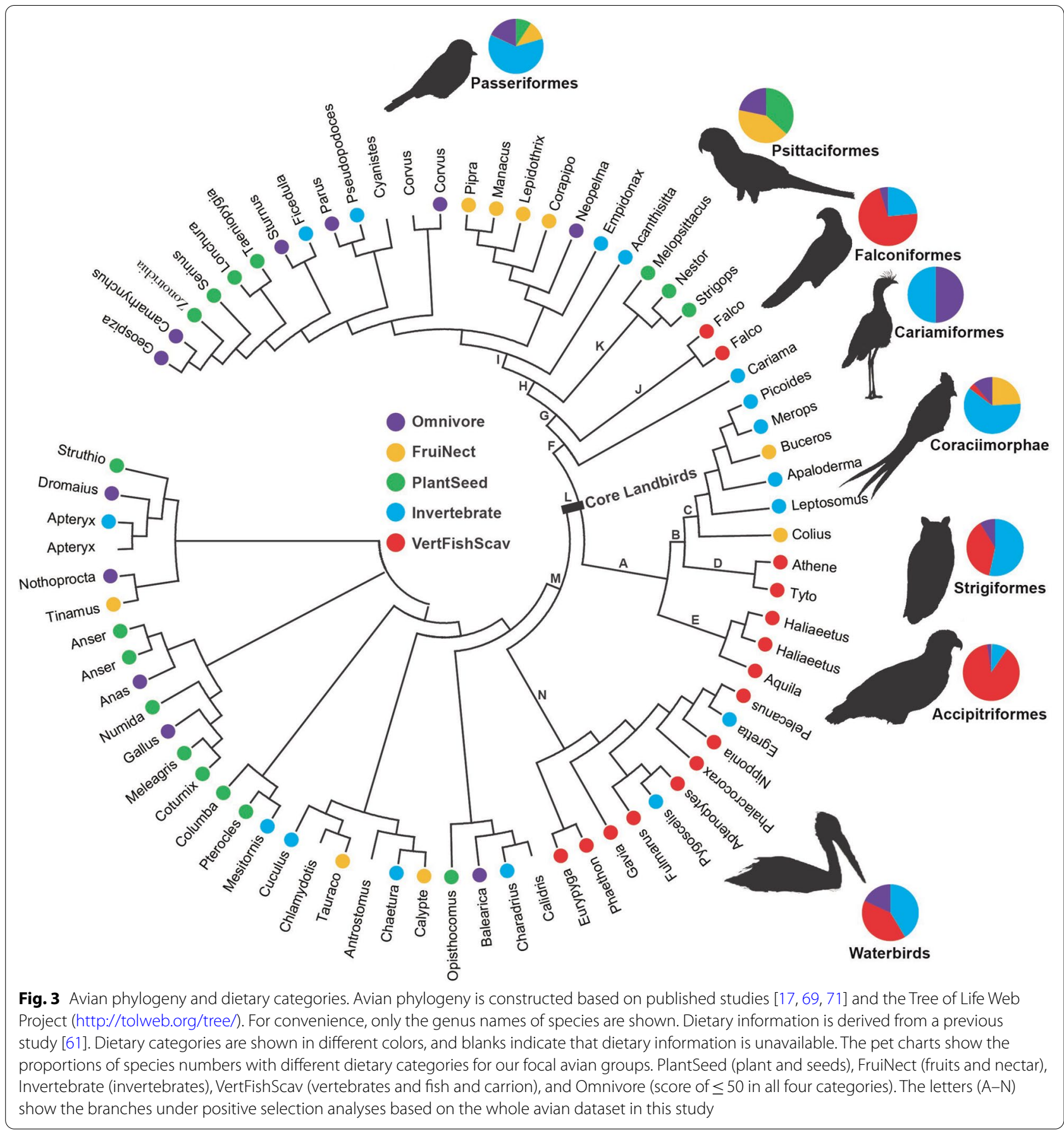

Our results showed that the common ancestor of core landbirds exhibited a marked intensified selection on the two genes (APOA4 and FABP1) related to fat digestion and absorption (Additional files 6, 9: Table S6, S9), with a much larger $\mathrm{K}$ value $(\mathrm{K} \approx 50, \mathrm{p}<0.05)$ than a neutrality indicator of $\mathrm{K}=1$. APOA4 encodes apolipoprotein A4 and is a potent activator of lecithincholesterol acyltransferase. FABP1 encodes the fatty acid binding protein found in liver and plays a role in fatty acid uptake, transport, and metabolism (Additional file 6: Table S6). We also found that another two fat digestion and absorption-related genes (AGPAT1 and $C D 36)$ showed a relatively slight selection relaxation, with $\mathrm{K}$ values of 0.95 and 0.89 , respectively. Moreover, we found three carbohydrate digestion and absorption-related genes (HK3, PIK3CD, and $S I)$ were 
under an intensified selection, and two protein digestion and absorption-related genes (MME and SLC9A3) respectively showed a slightly intensified selection and a marked selection relaxation (Additional file 9: Table S9). It seems that the ancestral core landbirds were subject to a relatively intensified selection for fat and carbohydrate digestion and absorption, which was more similar to that found in owls (Additional files 4, 5, 6, 7: Tables S4-7).

Based on the whole avian dataset, we also conducted the positive selection analyses along the common ancestor branch of core landbirds, as well as along other branches of interest (branches A-N) (Fig. 3, Additional files 10, 11: Tables S10-11). No PSGs were found along the common ancestor branch of core landbirds, while we found that all three birds of prey harbored PSGs that are involved in fat digestion and absorption, and non-raptorial birds showed predominant positive selection on the genes involved in protein or carbohydrate digestion and absorption (Additional file 6: Table S6, Additional files 10, 11: Tables S10-11). Specifically, for the three birds of prey, two PSGs from each of them were found, with $M E P 1 B$ and PLA2G2E found in Strigiformes, CPB1 and $F A B P 1$ found in Accipitriformes, and G6PC2 and $A B C A 1$ found in Falconiformes. Among the six PSGs detected, PLA2G2E, FABP1, and ABCA1 are involved in fat digestion and absorption, while the other three are related to either protein or carbohydrate digestion and absorption (Additional file 11: Table S11). In addition to the raptors, we also found one PSG (SLC15A1) along the Coraciimorphae branch, and one PSG (G6PC3) along the Passerimorphae branch, and six PSGs (CELA3B, SLC15A1, $C T R L, H K 3, G 6 P C 3$, and $A P O B$ ) along Passeriformes branch. However, almost all of these PSGs found in the non-raptorial birds are involved in protein or carbohydrate digestion and absorption, with the exception of $A P O B$ (Additional file 11: Table S11). We also examined the positive selection along the branch leading to parrots (Psittaciformes), and strikingly, we found 8 PSGs. Among the eight PSGs, four (APOA4, APOB, CLPS, and $A B C G 5)$ are involved in fat digestion and absorption, three (MME, CPA2, and $P R C P)$ are involved in protein digestion and absorption, and one (HK3) is involved in carbohydrate digestion and absorption. Finally, we examined the positive selection along the common ancestor branch (branch $\mathrm{M}$ ) of core landbirds and their sister taxa, waterbirds (Fig. 3, Additional files 10, 11: Tables S10-11), and one gene, AGPAT1, which is involved in fat digestion and absorption (Additional file 6: Table S6), was found to be under positive selection. Taken together, our results based on both the core landbird dataset and the whole avian dataset consistently show that the three birds of prey exhibited an enhanced selection for fat digestion and absorption, while their non-raptorial relatives tend to exhibit an enhanced selection for protein and/or carbohydrate digestion and absorption.

\section{Discussion}

We in this study used target enrichment sequencing to obtain the coding sequences of 308 genes to examine the molecular bases that underlie the ecological diversification of core landbirds in the context of the MPE approach. Our results showed that different core landbird taxa exhibit evolutionary enhancements in different aspects, suggesting lineage-specific selection. For instance, for owls, which are mainly crepuscularly and nocturnally active and are well known to have acute hearing [25], we detected evidence of the positive selection of five vision genes that mainly contribute to dim-light vision (Fig. 2, Additional file 5: Table S5) and their absence of the SWS1 sequence, suggesting enhanced dim-light vision and decreased bright-light vision, consistent with previous studies $[5,9,16]$. Besides their enhanced dim-light vision, we also found evidence of the positive selection of two hearing-related genes, COL4A5 and COL4A6, in owls (Additional file 5: Table S5). The two genes play a critical role in formation of basement membranes (Fig. 2), which are responsible for transferring sound waves to the organ of Corti for the sense of hearing. The positive selection of the two hearing-related genes in owls may suggest their increased sensitivity to sound in dim-light conditions. Moreover, we also detected evidence of the positive selection of one cold-sensor gene, TRPA1, in owls (Additional file 5: Table S5), which may be useful for them to sense low ambient temperatures at night. Unlike owls, for two diurnal taxa, Coraciimorphae and Passeriformes, we found almost solely cone-expressed vision genes, which are involved in photoresponse and photoresponse recovery, to be under positive selection (Additional files 4, 5: Tables S4-5), suggesting their increased visual acuity and promoted capacity for motion detection in bright-light conditions. In addition to their promoted bright-light vision, we also detected their predominant positive selections on hearing-related genes, suggesting that they may have evolved enhanced hearing as well. Many members of Coraciimorphae and Passeriformes are arboreal and are very vocal [4], and a promoted bright-light vision and enhanced hearing may help in the avoidance of obstacles and facilitating vocal communication in forest environments. We also detected the positive selection of language-related genes in owls and falcons, and particularly, we found the positive selection of the FOXP2 gene, a well-known language gene, in the common ancestor of falcons and Passerimorphae (Additional file 5: Table S5), suggesting their evolutionary modification of language. 
Our results demonstrated that all three birds of prey convergently evolved an enhanced capability for fat digestion and absorption, while non-raptorial birds tend to exhibit a promoted evolutionary enhancement for protein and carbohydrate digestion and absorption (Additional files 6, 7: Tables S6-7, Additional files 10, 11: Tables S10-11). Consistent with our finding of an increased capability for fat utilization in birds of prey, one recent study shows the relatively accelerated evolution of one lipid metabolism-related gene, PPARA, across birds of prey [5]. Previous studies show that the digestive physiology of animals evolves in parallel with their diets [26], and their digestion enzyme activities are known to be positively correlated with the amount of protein, carbohydrate, and lipid intake [27]. Our finding of an enhanced capability for fat utilization in birds of prey may be associated with their specialized carnivorous diets. All three birds of prey consume a considerable amount of vertebrates as prey (Fig. 3), which are relatively rich in protein and fat [28]. Fats contain the highest amounts of energy, with more than twice the number of calories as the same amount of proteins or carbohydrates [28]. An enhanced capability for fat utilization may help birds of prey to adapt to their relatively fat-rich diet. Unlike birds of prey, non-raptorial birds, such as Passeriformes and Coraciimorphae, eat a considerable amount of invertebrate as prey as well as plant food (e.g., seeds, fruits and nectar) (Fig. 3), which are generally protein-rich and carbohydrate-rich, respectively [28-30]. An increased capability for the utilization of protein and carbohydrates in nonraptorial birds may be partly attributed to the relative high proportion of protein and carbohydrates in their diets. Consistent with this, it has been known that most herbivores, from fishes to mammals, have a very high number of glucose transporters relative to carnivores, suggesting a relative evolutionary enhancement of carbohydrate utilization in herbivores [26].

Consistent with the enhanced capability of lipid utilization in birds of prey found in this study, previous genomic studies have well documented an enhanced lipid utilization in many carnivorous mammals (e.g., dog, cats, polar bear, cetaceans) [31-40], carnivorous reptiles (e.g., pythons, Komodo dragons) [41, 42] and even modern humans, such as the Maasai, who consume a fat-rich diet [43]. In addition to these findings at the molecular level, anatomically, carnivores are usually found to retain a gallbladder, and their gallbladders are relatively large, while for those animals that feed primarily on plant food, the gallbladder is absent or relatively small $[26,44]$. The gallbladder is used for storing bile, which is essential for the digestion of fat. The evolutionary enhancement of gallbladders in carnivorous animals may suggest a relatively high selection pressure for fat digestion, in line with the molecular findings mentioned above. Nevertheless, an enhanced lipid metabolism has also been reported in some ruminants (e.g., sheep, deer), which are typically herbivores. However, this is considered to be mainly due to their dependence on volatile fatty acids (VFAs) as an important nutrient source, which are generated by their rumen microbial flora from plant material $[45,46]$. It should be noted that we also detected a notable evolutionary enhancement of fat utilization-related genes along the branches leading to Passerimorphae (parrots to songbirds) and parrots, respectively (Additional files 7 , 11: Table S7, S11). We speculated that this may be partly attributed to the specific diet of parrots. Parrots largely include plant food, including nuts and seeds [47], in their diets (Fig. 3), and in particular, they have evolved a short, deep, hooked beak and a shortened, downward-curved lower jaw, which are adapted for cracking hard nuts and seeds [48], and nuts are generally high in fat [28]. One recent study shows that $37.68 \%$ of parrot species are known to ingest nuts in their diet, and these species are scattered across almost the whole parrot phylogeny, including the deepest and basal branches [47] (Additional file 12: Fig. S1). This may suggest that an increased capability of fat utilization may have evolved early accompanying the origin of parrots. These findings may suggest that the digestive system-related genes of animals may have been evolutionarily modified to adapt to the nutrient compositions (e.g., proteins, carbohydrates, and lipids) that they directly utilize in their diets.

The differentiated selections of digestive system-related genes between raptors and non-raptorial birds found in this study shed light on the origin of raptorial lifestyles in core landbirds. Core landbirds harbor three relatively distantly related birds of prey (Accipitriformes, Strigiformes and Falconiformes), and previous studies hypothesize that the common ancestor of core landbirds may be an apex predator [2, 3]. Our findings show that the common ancestor of core landbirds exhibited a particularly enhanced selection intensification for fat digestion and absorption (Additional file 9: Table S9). And this is similar with that observed in the three birds of prey, providing a relatively strong support for this hypothesis. However, we could not completely exclude the possibilities that the common ancestor of core landbirds could be of other types of dietary guilds, such as a plant eater that relies on a fat-rich plant food (e.g., nuts). However, other lines of evidence provide additional support for the common ancestor of core landbirds as an apex predator, as follows: (1) The three birds of prey are phylogenetically positioned at the deepest and/or basal branches within core landbirds [2, 3], suggesting their possibly early evolution. (2) Fossil evidence indicates that birds of prey (owls) occur earlier than almost all other members 
of core landbirds [49, 50]. (3) Our selection analyses demonstrate that Coraciimorphae showed a substantial selection relaxation of fat and protein digestion and absorption-related genes (Additional file 7: Table S7). This is consistent with their possible secondary loss of the raptorial lifestyle. (4) Previous studies demonstrate high transition rates from a carnivorous diet to other dietary guilds but less occurrences vice versa during the evolutionary histories of birds and mammals [51, 52]. This is consistent with the proposed raptorial lifestyle of ancestral core landbirds and their subsequent evolutionary transitions to the two non-raptor lineages leading to Passerimorphae and Coraciimorphae [2].

Core landbirds may have initially evolved in South America [53] as an apex predator, closely related to the extinct top predators, Phorusrhacidae, in the Cenozoic of South America [54, 55], and subsequently they dispersed globally and underwent an adaptive radiation to make use of different niches [53]. Accipitriformes hunt by day, Strigiformes hunt by night, while Falconiformes hunt by both day and night [16]. And two lineages, Passerimorphae and Coraciimorphae, may be secondarily derived from their raptorial ancestors and are mainly active during the day, as evidenced in this study, and evolved to exploit other non-raptorial diet niches (Fig. 3) $[2,54]$. Accumulated fossil evidence has shown that Eocene relatives of Coraciimorphae members (mousebirds and cuckoo-rollers) and Passerimorphae (parrots) have raptor-like feet or claws, and stem group passerines show parrot-like skeletal morphology $[2,56]$. These transitional fossils may suggest a gradual evolutionary change of Coraciimorphae and Passerimorphae from their raptorial-like ancestors [2]. If this is the case, we may speculate that the resemblance of parrots to birds of prey, such as their raptor-like curved beak, may be derived from their raptorial progenitors as well. It is noteworthy that our finding of the positive selection of one fat utilization-related gene, AGPAT1, along the common ancestor branch of core landbirds and their sister taxa, waterbirds, suggests the possibility of its raptorial lifestyle. And this is consistent with the considerable occurrences of carnivorous birds that prey on vertebrates in both core landbirds (e.g., birds of prey) and waterbirds (Fig. 3). This may imply that a raptorial lifestyle may have evolved much earlier than previously realized.

It should be noted that our selection analyses are based on the tree-like phylogenetic relationships of our focal taxa (Fig. 1), while recent studies suggest that reticulate events, for instance, hybridization, can lead to the deviations of phylogenetic relationships of taxa from tree-like patterns [57]. Previous studies suggest that living birds (including core landbirds) show relatively high levels of hybridization $[10,58]$, while its occurrence in ancestral bird lineages remains unknown. Therefore, in the future, examining the incidence of ancestral hybridization may be helpful to determine its possible effects on phylogenetic relationships of core landbirds. In addition, it has been reported that the exon capture success is negatively associated with phylogenetic distance of species [21], which may also affect our positive selection results. Finally, given that we in this study only used digestive system-related genes involved in three KEGG pathways as the molecular markers of diets, further studies involving much more digestive system-related genes will be helpful to determine the effects of dietary variation on the evolution of nutrient utilization-related genes.

\section{Conclusions}

Our study shows that core landbirds are subject to lineage-specific selections, and that different groups show evolutionary enhancements in different aspects. Despite this evolutionary specialization, two general evolutionary patterns emerge. First, nocturnal birds are characterized by an evolutionary enhancement in dim-light vision, while diurnal birds tend to evolve an enhanced bright-light vision, in line with previous studies [16]. More importantly, we find that the adaptive evolutions of digestive system-related genes are capable of reflecting the changes of nutrient compositions (e.g., proteins, carbohydrates, and lipids) in the diets of different avian taxa. This provides an important insight into reconstructing the ancestral dietary state of animals in the context of molecular phyloecology $[17,18]$. Particularly, our results based on two different datasets (core landbird dataset and whole avian dataset) consistently demonstrated that all three birds of prey show a convergent evolutionary enhancement for fat digestion and absorption, while non-raptorial birds tend to evolve an increased capability for protein and carbohydrates digestion and absorption, in relation to their differentiated diets. In light of this finding, our results provide relatively strong support for the raptorial lifestyle of ancestral core landbirds, implying a single origin of birds of prey, followed by two subsequently secondary losses in other non-raptorial birds. Moreover, our study implies that a raptorial lifestyle likely occurred much earlier than previously realized, possibly at least tracing back to the common ancestor of core landbirds and their sister taxa, waterbirds.

\section{Method}

\section{Datasets and taxa used}

In this study, we constructed two datasets, the core landbird dataset (Fig. 1) and the whole avian dataset (Fig. 3). In the core landbird dataset, the coding sequences of 78 core landbird species and a few outgroups were involved. Among the 78 species, 45 species were under 
target enrichment sequencing in this study, and the other 33 species' gene sequences were cited from GenBank. The 45 species under the target enrichment sequencing included 28 Strigiformes species, 13 Accipitriformes species, two Falconiformes species, one Coraciimorphae species and one Passeriformes species (Fig. 1). Particularly, the 28 species of Strigiformes were selected to represent all of its two known extant families (Tytonidae and Strigidae), five subfamilies, and nine tribes, with the only exception being Phodilinae [59]. The 13 species of Accipitriformes were from nine genera, two families (Cathartidae and Accipitridae), and particularly, 12 species of them were from Accipitridae and represented many of its divergent clades [60]. In addition to the core landbird dataset, which contained species from almost solely core landbirds, we also constructed the whole avian dataset, which contained the full-length coding gene sequences from all avian species (73 species in total) available from GenBank (Fig. 3). In total, 125 avian taxa across the core landbird dataset and whole avian dataset were used in this study.

\section{Diet dataset}

Avian diet information was used from one dataset, EltonTraits 1.0, which includes the compiled dietary information of a total of 9993 extant bird species from diverse published literature [61]. Accordingly, each species is assigned to the dominant among five diet categories: PlantSeed (plant and seeds), FruiNect (fruits and nectar), Invertebrate (invertebrates), VertFishScav (vertebrates and fish and carrion), and Omnivore (score of $\leq 50$ in all four categories). In addition to EltonTraits 1.0, we also referred to another parrot-specific dataset for its dietary analyses in the context of parrot phylogeny. This dataset includes a time-calibrated phylogenetic supertree of all 398 extant parrot species and detailed information about their diet components (e.g., seeds, nuts) [47].

\section{Gene list used}

The genes related to vision, hearing, language, temperature sensation, beak shape, taste transduction, and carbohydrate, protein and fat digestion and absorption were involved in this study (Additional file 1: Table S1). In total, 308 genes were included. These gene lists involved in the different biological functions were compiled from the published literature and/or online KEGG Pathway database (please see Additional file 13). Briefly, for vision, the 33 phototransduction genes that are used to determine diel activity patterns $[17,18]$ were used. For hearing, the 95 genes related to hearing were retrieved from the published literature. For temperature sensation, the 15 genes that are mainly involved in thermosensitive TRP channels spanning from sensing warm to cold temperatures were used based on the published literature. For language and beak shape, the related genes were mainly retrieved from the published literature. Finally, for taste transduction, and carbohydrate, protein and fat digestion and absorption, the gene lists were retrieved from the relevant KEGG pathways (maps 04742, 04973, 04974, 04975). The function descriptions of these genes were referred to the published literature and the KEGG pathway database (Additional file 13) as well as the Online Mendelian Inheritance in Man (OMIM) and/or Gene in NCBI.

\section{Bait design}

We designed baits mainly based on the coding sequences of 308 genes of three species from GenBank, including Falco cherrug, Haliaeetus albicilla, and Tyto alba, representing three birds of prey, Falconiformes, Accipitriformes, and Strigiformes, respectively. If their gene sequences were unavailable for a certain gene, the gene sequences from their phylogenetical relatives were used, including Falco peregrinus, Aquila chrysaetos, Haliaeetus leucocephalus, Picoides pubescens, Apaloderma vittatum, and Colius striatus. Moreover, we also included the transcript sequences of 33 phototransduction genes from 15 core landbird species published previously in our lab [16]. Based on these gene sequences, we designed 90nt probes with $2 \times$ tiling density, then we screened them against Falcon peregrinus genome with high-coverage [6] (GCF_000337955.1_F_peregrinus_v1.0_genomic. fna.gz) as well as the owl mitochondrial genome, and we applied an "ultrastringent" level of filtration to identify and remove those baits that were likely to hybridize and capture more than one genomic region, and eventually, 59,969 ultrastringent-baits were used for subsequent target enrichment via hybridization-based capture (MYcroarray, Arbor Biosciences, USA).

\section{Sample collection, DNA extraction and target enrichment sequencing}

We collected blood, muscle, and liver samples from 46 core landbird taxa (Additional file 2: Table S2), of which 27 blood samples were obtained in this study, 15 muscle samples were collected previously in our lab [16], and four liver samples were contributed by the Jilin Provincial Museum of Natural History. The 27 blood samples obtained in this study were collected onto FTA cards and then mailed to a laboratory and stored at $-80{ }^{\circ} \mathrm{C}$ condition. We abstracted genomic DNA from the blood samples using the DNeasy Blood \& Tissue Kit (QIAGEN), and we abstracted genomic DNA from the muscle or liver samples using the Ezup Column Animal Genomic DNA Purification Kit (Sangon Biotech, Beijing). DNA concentration was measured by 
NanoDrop 2000C (Thermo Scienfitic) (Additional file 2: Table S2).The experimental procedures were performed in accordance with an animal ethics approval granted by Northeast Normal University. All experimental procedures carried out in this study were approved by the National Animal Research Authority of Northeast Normal University, China (Approval Number: NENU20080416) and the Forestry Bureau of Jilin Province of China (Approval Number: [2006]178).

We quantified the DNA samples of the 46 taxa using the Quant-iT(TM) Picogreen(R) dsDNA Assay kit (ThermoFisher). The visualization of a subset of samples on a Bioanalyzer High-Sensitivity DNA Chip (Agilent) indicated that the DNA was predominantly high in molecular weight. Up to 4 ug of each sample was then sheared with a Qsonica Q800R instrument and selected to modal lengths of roughly 300nt using a dual-step SPRI bead cleanup. We then converted on average $384 \mathrm{ng}$ of the sheared, size-selected samples to Illumina $(R)$ sequencing libraries using chemistry based on the KAPA HyperPrep(TM) (Kapa Biosystems) kit for Illumina. After ligation, the libraries were amplified using KAPA HiFi HotStart ReadyMix (Kapa Biosystems) for 6,8 or 10 cycles, inversely scaled to the mass taken to the library preparation, using the manufacturer's recommended thermal profile. Custom i7 and i5 $8 \mathrm{nt}$ indices were incorporated during this amplification in combinations designed to prevent cross-indexing during post-capture pooled amplification. After purification with SPRI beads, we then quantified each indexamplified library using the Quant-iT(TM) Picogreen(R) dsDNA Assay kit (ThermoFisher). For target enrichment, two samples (Corvus corax and Upupa epops) were captured individually using $500 \mathrm{ng}$ total library per reaction. All other samples were captured in pools of eight samples, using $80 \%$ of the available library mass up to $100 \mathrm{ng}$ each. Enrichment followed the myBaits Manual version 3 using double the block \#3 reagent and $65{ }^{\circ} \mathrm{C}$ temperatures for all relevant steps. After enrichment, half of the cleaned bead suspension was taken to 10 cycle amplification using universal primers and KAPA HiFi HotStart ReadyMix (Kapa Biosystems). After purification, each captured library was quantified with quantitative PCR and combined in equimolar ratios prior to sequencing. The equilibrated sequencing pool was then submitted to the Genomic Services Lab at Hudson Alpha (Huntsville, Alabama, USA) for paired-end 125 bp sequencing on a HiSeq 2500 instrument using version 4 chemistry. Raw FASTQs were delivered following the standard CASAVA post-processing pipeline, and were deposited in the Sequence Read Archive (SRA) database with accession numbers (SRR11449714-59).

\section{Sequence assembly and alignment}

Raw reads were processed by SOAPnuke (version 1.5.6, https://github.com/BGI-flexlab/SOAPnuke) to remove adapter sequences and low quality reads with the following parameters: $-\mathrm{n} \quad 0.01-\mathrm{l} \quad 20$-q 0.4 - $\mathrm{Q}$ 2. Then, duplicate reads were filtered using a custom Perl script. Prior to assembly, all clean reads were aligned to our target sequences using BLASTN to identify intronexon boundaries (e value cutoff $=1 \mathrm{e}^{-5}$ ), and then intron sequences were trimmed. All processed reads were de novo assembled into contigs by Trinity [62] with the following parameters: -min_kmer_cov 3 -min_glue 3 -bfly_opts '-V 5 -edge-thr $=0.1$-stderr' - min_contig_length 150 . Then all contigs were aligned to our target gene sequences originally used for bait design using the BLASTP with default parameters. And we chose hits with an identity $>80 \%$ and coverage $>60 \%$ as the candidate contigs that were associated with certain targets (intarget assemblies). Subsequently, these candidate contigs were joined together with Ns based on their relative BLAST hit positions in regard to the target sequences. To verify the orthology of these candidate contigs, they were then blasted against the National Center for Biotechnology Information (NCBI) nr/nt database using BLASTN online. We kept for subsequent analyses only those candidate contigs with their best hit being our target gene; otherwise, that particular sequence was discarded. Finally, sequencing coverage and sequencing depth were evaluated by BWA [63] with a default parameter to evaluate capture efficiency. Gene sequences were aligned using webPRANK (http://www.ebi.ac.uk/goldman-srv/webpr ank/), and individual sequences with low identities, long indels, multiple ambiguous bases Ns, and/or too short a length were removed. After this pruning, high-quality alignments were constructed, and their translated protein sequences were blasted against the non-redundant protein sequence database to confirm the correctness of sequence cutting.

\section{Positive selection analyses}

We used the branch and branch-site models implemented in the Codeml program of PAML [23] for our positive selection analyses. For this, we constructed unrooted species trees following previous studies [2, 17, 59, 60, 64-73] and the Tree of Life Web Project (http:// tolweb.org/tree/). We used a codon-based maximumlikelihood method to evaluate the ratio of non-synonymous to synonymous substitutions per site $(\mathrm{dN} / \mathrm{dS}$ or $\omega$ ), and used likelihood ratio tests (LRT) to determine statistical significance. A statistically significant value of $\omega>1$ suggests a positive selection. Specifically, for the branch model analyses, we used a two-rate branch model to detect possible positive selection signals along 
a particular branch. With the analyses, our focal branches were respectively labeled foreground branches, and others were used as background branches. The two-rate branch model was then compared with the one-rate branch model to determine statistical significance using LRT. If a statistically significant value of $\omega>1$ in a foreground branch was found, the two-ratio branch model was then compared with the two-ratio branch model with a constraint of $\omega=1$ in the foreground branch to further examine whether the $\omega>1$ of the foreground branch was supported with statistical significance. In addition to the branch model, we also used a branch-site model (Test 2) to detect positively selected sites along our focal branches (foreground branches). Test 2 compares a modified model A with its corresponding null model with $\omega=1$ constrained to determine the statistical significance using LRT. The empirical Bayes method was used to identify positively selected sites. For our result robustness, we further examined the dependency of our positive selection results on the initial value variations of kappa and omega. For this, we respectively used two different initial values of kappa (kappa $=0.5,3.0)$ and of omega $(\omega=0.5,2.0)$ for our positive selection analyses, and in total, four independent runs were conducted for each of the PSGs found.

\section{Selection intensity analyses}

We evaluated the relative selection intensity changes of genes using RELAX [24], which is available from the Datamonkey webserver (http://test.datamonkey. org/relax). The relative selection intensity of genes was measured using a parameter $\mathrm{k}$ value. The $\mathrm{k}$ value and its statistical significance were evaluated given a priori partitioning of test branches and reference branches in a codon-based phylogenetic framework. $\mathrm{K}>1$ shows an intensified selection, and $\mathrm{k}<1$ shows a relaxed selection. Intensified selection is expected to exhibit $\omega$ categories away from neutrality $(\omega=1)$, while relaxed selection is expected to exhibit $\omega$ categories converging to neutrality $(\omega=1)$. Statistical significance was evaluated by comparing an alternative model to a null model using LRT. The alternative model assumes different $\omega$ distributions of the test and reference branches, while the null model assumes $\mathrm{k}=1$ and the same $\omega$ distribution of both test and reference branches.

\section{Supplementary Information}

The online version contains supplementary material available at https://doi. org/10.1186/s12862-021-01888-5.

Additional file 1: Table S1. Gene list used in this study.
Additional file 2: Table S2. 46 core landbird taxa under gene capture in this study.

Additional file 3: Table S3. Gene sequences cited from GenBank.

Additional file 4: Table S4. Positively selected genes identified by branch model based on the core landbird dataset.

Additional file 5: Table S5. Positively selected genes identified by branch-site model based on the core landbird dataset.

Additional file 6: Table S6 Function description of digestive systemrelated genes under adaptive evolution. Function description of genes is referred to NCBI Gene and/or OMIM and Kyoto Encyclopedia of Genes and Genomes (KEGG).

Additional file 7: Table S7. The genes under relatively relaxed selection $(k<1)$ and relatively intensified selection $(k>1)$ of our focal taxa (test branch) relative to the common ancestors of our focal taxa and their sister taxa (reference branch). This result is based on the core landbird dataset.

Additional file 8: Table S8. Digestive system gene sequences of Avian taxa cited from GenBank.

Additional file 9: Table S9. The genes under relatively relaxed selection $(k<1)$ and relatively intensified selection $(k>1)$ along the common ancestor branch (test branch) of core landbirds relative to the common ancestor branch (reference branch) of core landbirds and their sister taxa, waterbirds. This result is based on the whole avian dataset.

Additional file 10: Table S10. Positively selected genes identified by branch model based on the whole avian dataset.

Additional file 11: Table S11. Positively selected genes identified by branch site model based on the whole avian dataset.

Additional file 12: Fig. S1 Phylogenetic supertree of 413 parrot species. Parrots that consume nuts in their diets are shown in red. The phylogenetic supertree and dietary information follow one published study (Burgio et al. [47])

Additional file 13. Literature sources of the gene list used in this study.

\section{Acknowledgements}

We acknowledge Michael Wink, David Brinker, Stacy Campopiano, Dejeanne Doublet, Caroline Ebinger, Bob Fox, Rebecca Gordon, George V. Kollias Jr., Brenda H. Little, Dennis D. Little, Sharon Matola, Julia M. Mello, Dave Oleyar, Noelle Ronan, Annik Gionet Rollick, Louise Schimmel, Russell Thorstrom, Bob Tompkins, Lynn Tompkins, Colleen Wisinski, Yu Liu, Wei Liang, Haitao Wang for helping sample collections.

\section{Authors' contributions}

YW conceived the study, performed experiments, conducted data analyses, and wrote the paper. DHJ conceived the study, collected blood samples and read the manuscript. SW helped for sequence assembly and sequence abstraction. YY, YZ and LG helped for selection analyses. All authors read and approved the final manuscript.

\section{Funding}

This research was supported by the National Natural Science Foundation of China (Grant Numbers, 32171604, 31770401) and the Fundamental Research Funds for the Central Universities.

\section{Availability of data and materials}

All the sequences reported in this study were deposited in the Sequence Read Archive (SRA) database with accession numbers (SRR1 1449714-59).

\section{Declarations}

\section{Ethics approval and consent to participate}

The experimental procedures were performed in accordance with an animal ethics approval granted by Northeast Normal University. All experimental procedures carried out in this study were approved by the National Animal Research Authority of Northeast Normal University, China (Approval Number: 
NENU-20080416) and the Forestry Bureau of Jilin Province of China (Approval Number: [2006]178). No animals were killed or captured in this study.

\section{Consent for publication}

Not applicable.

\section{Competing interests}

We have no competing interests to declare.

\section{Author details}

${ }^{1}$ School of Life Sciences, Northeast Normal University, Changchun 130024 China. ${ }^{2}$ Bio-Intelligence Co. Ltd, Shenzhen 518000, China. ${ }^{3} \mathrm{Global}$ Owl Project, 6504 Carriage Drive, Alexandria, VA 22310, USA.

\section{Received: 28 August 2020 Accepted: 10 August 2021} Published online: 28 August 2021

\section{References}

1. Hackett SJ, Kimball RT, Reddy S, Bowie RC, Braun EL, Braun MJ, Chojnowski JL, Cox WA, Han K-L, Harshman J, et al. A phylogenomic study of birds reveals their evolutionary history. Science. 2008;320:1763-8.

2. Jarvis ED, Mirarab S, Aberer AJ, Li B, Houde P, Li C, Ho SY, Faircloth BC, Nabholz B, Howard JT, et al. Whole-genome analyses resolve early branches in the tree of life of modern birds. Science. 2014;346:1320-31.

3. Prum RO, Berv JS, Dornburg A, Field DJ, Townsend JP, Lemmon EM, Lemmon AR. A comprehensive phylogeny of birds (Aves) using targeted next-generation DNA sequencing. Nature. 2015;526:569-73.

4. Del Hoyo J, Elliott A, Sargatal J. Handbook of the birds of the world, vol 1-16 Barcelona: Lynx Edicions; 1992-2011.

5. Cho YS, Jun JH, Kim JA, Kim H-M, Chung O, Kang S-G, Park J-Y, Kim H-J, Kim S, Kim H-J, et al. Raptor genomes reveal evolutionary signatures of predatory and nocturnal lifestyles. Genome Biol. 2019;20:181.

6. Zhang G, Li C, Li Q, Li B, Larkin D, Lee C, Storz J, Antunes A, Greenwold $M$, Meredith $R$, et al. Comparative genomics reveals insights into avian genome evolution and adaptation. Science. 2014;346:1311-20.

7. Chung O, Jin S, Cho YS, Lim J, Kim H, Jho S, Kim H-M, Jun J, Lee H, Chon A, et al. The first whole genome and transcriptome of the cinereous vulture reveals adaptation in the gastric and immune defense systems and possible convergent evolution between the Old and New World vultures. Genome Biol. 2015:16:215.

8. Hanna ZR, Henderson JB, Wall JD, Emerling CA, Fuchs J, Runckel C, Mindell DP, Bowie RC, DeRisi JL, Dumbacher JP. Northern spotted owl (Strix occidentalis caurina) genome: divergence with the barred owl (Strix varia) and characterization of light-associated genes. Genome Biol Evol. 2017;9:2522-45

9. Höglund J, Mitkus M, Olsson P, Lind O, Drews A, Bloch NI, Kelber A, Strandh M. Owls lack UV-sensitive cone opsin and red oil droplets, but see UV light at night: retinal transcriptomes and ocular media transmittance. Vision Res. 2019;158:109-19.

10. Lamichhaney S, Berglund J, Almén MS, Maqbool K, Grabherr M, MartinezBarrio A, Promerová M, Rubin C-J, Wang C, Zamani N, et al. Evolution of Darwin's finches and their beaks revealed by genome sequencing. Nature. 2015;518:371-5.

11. Wirthlin M, Lima N, Guedes R, Soares A, Almeida L, Cavaleiro N, de Morais LG, Chaves A, Howard J, Teixeira M, et al. Parrot genomes and the evolution of heightened longevity and cognition. Curr Biol. 2018;28:4001-8.

12. Zhan X, Pan S, Wang J, Dixon A, He J, Muller MG, Ni P, Hu L, Liu Y, Hou H, et al. Peregrine and saker falcon genome sequences provide insights into evolution of a predatory lifestyle. Nat Genet. 2013;45:563-6.

13. Zhou C, Jin J, Peng C, Wen Q, Wang G, Wei W, Jiang X, Price M, Cui K, Meng $Y$, et al. Comparative genomics sheds light on the predatory lifestyle of accipitrids and owls. Sci Rep. 2019;9:2249.

14. Borges R, Khan I, Johnson WE, Gilbert MTP, Zhang G, Jarvis ED, O'Brien SJ, Antunes A. Gene loss, adaptive evolution and the co-evolution of plumage coloration genes with opsins in birds. BMC Genomics. 2015;16:751.

15. Chen $\mathrm{Y}-\mathrm{H}$, Zhao H. Evolution of digestive enzymes and dietary diversification in birds. Peer J. 2019;7:e6840.
16. Wu Y, Hadly EA, Teng W, Hao Y, Liang W, Liu Y, Wang H. Retinal transcriptome sequencing sheds light on the adaptation to nocturnal and diurnal lifestyles in raptors. Sci Rep. 2016;6:33578.

17. Wu Y, Wang H. Convergent evolution of bird-mammal shared characteristics for adapting to nocturnality. Proc Royal Soc B. 2019;286:20182185.

18. Wu Y, Wang H, Hadly EA. Invasion of ancestral mammals into dim-light environments inferred from adaptive evolution of the phototransduction genes. Sci Rep. 2017;7:46542.

19. Grover CE, Salmon A, Wendel JF. Targeted sequence capture as a powerful tool for evolutionary analysis. Am J Bot. 2012;99:312-9.

20. Jones MR, Good JM. Targeted capture in evolutionary and ecological genomics. Mol Ecol. 2016;25:185-202.

21. Portik DM, Smith LL, Bi K. An evaluation of transcriptome-based exon capture for frog phylogenomics across multiple scales of divergence (Class: Amphibia, Order: Anura). Mol Ecol Resour. 2016;16:1069-83.

22. Schott RK, Panesar B, Card DC, Preston M, Castoe TA, Chang BS. Targeted capture of complete coding regions across divergent species. Genome Biol Evol. 2017;9:398-414.

23. Yang Z. PAML 4: phylogenetic analysis by maximum likelihood. Mol Biol Evol. 2007:24:1586-91.

24. Wertheim JO, Murrell B, Smith MD, Kosakovsky Pond SL, Scheffler K. RELAX: detecting relaxed selection in a phylogenetic framework. Mol Biol Evol. 2015;32:820-32.

25. König C, Weick F. Owls of the World. London: Christopher Helm; 2008.

26. Miller S, Harley J. Zoology. New York: McGraw-Hill Education; 2016.

27. Corring T. The adaptation of digestive enzymes to the diet: its physiological significance. Reprod Nutr Dévelop. 1980;20:1217-35.

28. Clinic M. Encyclopedia of foods: a guide to healthy nutrition. San Diego: Academic Press; 2002

29. Kim T-K, Yong HI, Kim Y-B, Kim H-W, Choi Y-S. Edible insects as a protein source: a review of public perception, processing technology, and research trends. Food Sci Anim Resour. 2019;39:521-40.

30. Raubenheimer D, Rothman JM. Nutritional ecology of entomophagy in humans and other primates. Annu Rev Entomol. 2013;58:141-60.

31. Cho YS, Hu L, Hou H, Lee H, Xu J, Kwon S, Oh S, Kim H-M, Jho S, Kim S, et al. The tiger genome and comparative analysis with lion and snow leopard genomes. Nat Commun. 2013;4:2433.

32. Endo Y, Kamei K, Inoue-Murayama M. Genetic signatures of lipid metabolism evolution in Cetacea since the divergence from terrestrial ancestor. J Evol Biol. 2018;31:1655-65.

33. Freedman AH, Schweizer RM, Ortega-Del Vecchyo D, Han E, Davis BW, Gronau I, Silva PM, Galaverni M, Fan Z, Marx P, et al. Demographicallybased evaluation of genomic regions under selection in domestic dogs. PLoS Genet. 2016;12:e1005851.

34. Kim S, Cho YS, Kim H-M, Chung O, Kim H, Jho S, Seomun H, Kim J, Bang WY, Kim C, et al. Comparison of carnivore, omnivore, and herbivore mammalian genomes with a new leopard assembly. Genome Biol. 2016;17:211.

35. Liu S, Lorenzen ED, Fumagalli M, Li B, Harris K, Xiong Z, Zhou L, Korneliussen TS, Somel M, Babbitt C, et al. Population genomics reveal recent speciation and rapid evolutionary adaptation in polar bears. Cell. 2014;157:785-94.

36. McGowen MR, Grossman LI, Wildman DE. Dolphin genome provides evidence for adaptive evolution of nervous system genes and a molecular rate slowdown. Proc Biol Sci. 2012;279:3643-51.

37. Montague MJ, Li G, Gandolfi B, Khan R, Aken BL, Searle SM, Minx P, Hillier LW, Koboldt DC, Davis BW, et al. Comparative analysis of the domestic cat genome reveals genetic signatures underlying feline biology and domestication. Proc Natl Acad Sci U S A. 2014;111:17230-5.

38. Tsagkogeorga G, Mcgowen MR, Davies KT, Jarman S, Polanowski A, Bertelsen MF, Rossiter SJ. A phylogenomic analysis of the role and timing of molecular adaptation in the aquatic transition of cetartiodactyl mammals. R Soc Open Sci. 2015;2:150156.

39. Wang Z, Chen Z, Xu S, Ren W, Zhou K, Yang G. 'Obesity'is healthy for cetaceans? Evidence from pervasive positive selection in genes related to triacylglycerol metabolism. Sci Rep. 2015;5:14187.

40. Wang Z, Xu S, Du K, Huang F, Chen Z, Zhou K, Ren W, Yang G. Evolution of digestive enzymes and RNASE1 provides insights into dietary switch of cetaceans. Mol Biol Evol. 2016;33:3144-57.

41. Castoe TA, de Koning AJ, Hall KT, Card DC, Schield DR, Fujita MK, Ruggiero RP, Degner JF, Daza JM, Gu W, et al. The Burmese python genome reveals 
the molecular basis for extreme adaptation in snakes. Proc Natl Acad Sci U S A. 2013;110:20645-50.

42. Lind AL, Lai YY, Mostovoy Y, Holloway AK, lannucci A, Mak AC, Fondi M, Orlandini V, Eckalbar WL, Milan M, et al. Genome of the Komodo dragon reveals adaptations in the cardiovascular and chemosensory systems of monitor lizards. Nat Ecol Evol. 2019;3:1241-52.

43. Wagh K, Bhatia A, Alexe G, Reddy A, Ravikumar V, Seiler M, Boemo M, Yao M, Cronk L, Naqvi A, et al. Lactase persistence and lipid pathway selection in the Maasai. PloS One. 2012;7:e44751.

44. Oldham-Ott CK, Gilloteaux J. Comparative morphology of the gallbladder and biliary tract in vertebrates: variation in structure, homology in function and gallstones. Microsc Res Tech. 1997;38:571-97.

45. Chen L, Qiu Q, Jiang Y, Wang K, Lin Z, Li Z, Bibi F, Yang Y, Wang J, Nie W, et al. Large-scale ruminant genome sequencing provides insights into their evolution and distinct traits. Science. 2019;364:eaav6202.

46. Jiang Y, Xie M, Chen W, Talbot R, Maddox JF, Faraut T, Wu C, Muzny DM, Li $Y$, Zhang $W$, et al. The sheep genome illuminates biology of the rumen and lipid metabolism. Science. 2014;344:1168-73.

47. Burgio KR, Davis KE, Dreiss LM, Cisneros LM, Klingbeil BT, Presley SJ, Willig MR. Phylogenetic supertree and functional trait database for all extant parrots. Data Brief. 2019;24:103882.

48. Benton MJ. Vertebrate palaeontology. Oxford: Wiley Blackwell; 2014.

49. Ksepka DT, Stidham TA, Williamson TE. Early Paleocene landbird supports rapid phylogenetic and morphological diversification of crown birds after the K-Pg mass extinction. Proc Natl Acad Sci U S A. 2017;114:8047-52.

50. Mayr G. The origins of crown group birds: molecules and fossils. Palaeontology. 2014;57:231-42.

51. Burin G, Kissling WD, Guimarães PR, Şekercioğlu ÇH, Quental TB. Omnivory in birds is a macroevolutionary sink. Nat Commun. 2016;7:11250.

52. Price SA, Hopkins SS, Smith KK, Roth VL. Tempo of trophic evolution and its impact on mammalian diversification. Proc Natl Acad Sci U S A. 2012;109:7008-12.

53. Claramunt S, Cracraft J. A new time tree reveals Earth history's imprint on the evolution of modern birds. Sci Adv. 2015;1:e1501005.

54. Brusatte SL, O'Connor JK, Jarvis ED. The origin and diversification of birds. Curr Biol. 2015;25:R888-98.

55. Mayr G. Paleogene fossil birds. Heidelberg: Springer; 2009.

56. Mayr G. Avian evolution: the Fossil Record of Birds and Its Paleobiological Significance. West Sussex: Wiley; 2016.

57. Ottenburghs J, van Hooft P, van Wieren SE, Ydenberg RC, Prins HH. Birds in a bush: toward an avian phylogenetic network. Auk. 2016;133:577-82.

58. Ottenburghs J, Ydenberg RC, Van Hooft P, Van Wieren SE, Prins HH. The Avian Hybrids Project: gathering the scientific literature on avian hybridization. Ibis. 2015;157:892-4.

59. Wink M, El-Sayed A-A, Sauer-Gürth H, Gonzalez J. Molecular phylogeny of owls (Strigiformes) inferred from DNA sequences of the mitochondrial cytochrome $b$ and the nuclear RAG-1 gene. Ardea. 2009;97:581-91.

60. Lerner HR, Mindell DP. Phylogeny of eagles, Old World vultures, and other Accipitridae based on nuclear and mitochondrial DNA. Mol Phylogenet Evol. 2005;37:327-46.
61. Wilman H, Belmaker J, Simpson J, de la Rosa C, Rivadeneira MM, Jetz W. EltonTraits 10: species-level foraging attributes of the world's birds and mammals: ecological Archives E095-178. Ecology. 2014;95:2027-2027.

62. Grabherr MG, Haas BJ, Yassour M, Levin JZ, Thompson DA, Amit I, Adiconis $X$, Fan L, Raychowdhury R, Zeng Q, et al. Full-length transcriptome assembly from RNA-Seq data without a reference genome. Nat Biotechnol. 2011;29:644-52.

63. Li H, Durbin R. Fast and accurate short read alignment with BurrowsWheeler transform. Bioinformatics. 2009:25:1754-60.

64. Dantas SM, Weckstein JD, Bates JM, Krabbe NK, Cadena CD, Robbins $M B$, Valderrama E, Aleixo A. Molecular systematics of the New World screech-owls (Megascops: Aves, Strigidae): Biogeographic and taxonomic implications. Mol Phylogenet Evol. 2016;94:626-34.

65. Fuchs J, Johnson JA, Mindell DP. Rapid diversification of falcons (Aves: Falconidae) due to expansion of open habitats in the Late Miocene. Mol Phylogenet Evol. 2015;82:166-82.

66. Haring E, Däubl B, Pinsker W, Kryukov A, Gamauf A. Genetic divergences and intraspecific variation in corvids of the genus Corvus (Aves: Passeriformes: (orvidae)-a first survey based on museum specimens. J Zool Syst Evol Res. 2012;50:230-46.

67. Johansson US, Ekman J, Bowie RC, Halvarsson P, Ohlson JI, Price TD, Ericson PG. A complete multilocus species phylogeny of the tits and chickadees (Aves: Paridae). Mol Phylogenet Evol. 2013;69:852-60.

68. Johnsen A, Kearns AM, Omland KE, Anmarkrud JA. Sequencing of the complete mitochondrial genome of the common raven Corvus corax (Aves: Corvidae) confirms mitogenome-wide deep lineages and a paraphyletic relationship with the Chihuahuan raven C. cryptoleucus. PloS One. 2017;12:0187316.

69. Jønsson KA, Fjeldså J. A phylogenetic supertree of oscine passerine birds (Aves: Passeri). Zool Scr. 2006;35:149-86.

70. Kriegs JO, Matzke A, Churakov G, Kuritzin A, Mayr G, Brosius J, Schmitz J. Waves of genomic hitchhikers shed light on the evolution of gamebirds (Aves: Galliformes). BMC Evol Biol. 2007;7:190.

71. McKay BD, Barker FK, Mays HL Jr, Doucet SM, Hill GE. A molecular phylogenetic hypothesis for the manakins (Aves: Pipridae). Mol Phylogenet Evol. 2010;55:733-7.

72. Riesing MJ, Kruckenhauser L, Gamauf A, Haring E. Molecular phylogeny of the genus Buteo (Aves: Accipitridae) based on mitochondrial marker sequences. Mol Phylogenet Evol. 2003;27:328-42.

73. Wright TF, Schirtzinger EE, Matsumoto T, Eberhard JR, Graves GR, Sanchez JJ, Capelli S, Müller H, Scharpegge J, Chambers GK, et al. A multilocus molecular phylogeny of the parrots (Psittaciformes): support for a Gondwanan origin during the Cretaceous. Mol Biol Evol. 2008;25:2141-56.

\section{Publisher's Note}

Springer Nature remains neutral with regard to jurisdictional claims in published maps and institutional affiliations.

\footnotetext{
Ready to submit your research? Choose BMC and benefit from:

- fast, convenient online submission

- thorough peer review by experienced researchers in your field

- rapid publication on acceptance

- support for research data, including large and complex data types

- gold Open Access which fosters wider collaboration and increased citations

- maximum visibility for your research: over $100 \mathrm{M}$ website views per year
}

At BMC, research is always in progress.

Learn more biomedcentral.com/submissions 